\title{
Política de Ciencia y Tecnología con Perspectiva de Género en México*
}

\author{
Norma BLAZQUEZ GRAF** \\ Lourdes Elena FERNÁNDEZ RIUS***
}

\section{Resumen}

El tema de género e igualdad en la investigación científica impulsa acciones de transformación y define un nuevo modelo para eliminar las relaciones de poder que subordinan a las mujeres. En este texto se propone visibilizar estos hechos y su significación, así como alentar la revisión acerca de una política de ciencia y tecnología con perspectiva de género que garantice el camino de la transformación.

Palabras clave: Género y Ciencia, Política Científica, Política Científica y Género.

* Recibido para publicación el 7 de marzo de 2016, aceptado el 10 de noviembre de 2016.

** Centro de Investigaciones Interdisciplinarias en Ciencias y Humanidades. Universidad Nacional Autónoma de México/ Red Mexicana de Ciencia, Tecnología y Género. blazquez@unam.mx

*** Instituto Universitario Internacional de Toluca/ Red Mexicana de Ciencia, Tecnología y Género. lulufrius2004@yahoo.com.mx 
Policy Review on Science and Technology with Gender Perspective In Mexico

\begin{abstract}
Gender and equality in scientific research drives transformation actions and defines a new model for eliminating power relations that subordinate women. In this paper it is proposed to bring these facts and their significance, as well as encouraging a policy review on science and technology with gender perspective to ensure the path of transformation.
\end{abstract}

Keywords: Gender and Science; Science Policy; Science Policy and Gender. 


\section{Introducción}

Los avances en la ciencia impactan el desarrollo de la economía, la cultura, la educación, el arte, la recreación y la salud. Actualmente predomina una visión integral del desarrollo de las ciencias que articula la protección e incremento de los recursos naturales, humanos, los conocimientos y tecnologías ancestrales, el ambiente y la historia, emergiendo cada vez con más fuerza la noción de una ciencia "situada" en la cual los valores epistémicos se vinculan con valores éticos que hacen insostenible la idea de una ciencia "neutral"

En paralelo, la incidencia del feminismo en el siglo XX y en la primera parte del siglo XXI se ha manifestado tanto en forma jurídica, como social y culturalmente, evidenciándose cada vez mayor integración y liderazgo mundial de las mujeres, las cuales han avanzado en casi todas las esferas de la actividad humana, lográndose oportunidades y derechos de participación cada vez mayores.

En este escenario surge uno de los sucesos más innovadores de las sociedades contemporáneas, el acceso creciente de las mujeres al saber de más alto nivel, la acreditación de ese saber y el ascenso de las mismas a puestos de toma de decisiones en el ámbito académico, aunque siguen existiendo barreras que les impiden participar en condiciones de igualdad en relación con los hombres.

Estas inequidades se manifiestan en la existencia de una segregación horizontal, traducida en la escasa presencia femenina en algunas ramas del conocimiento y en la existencia de una segregación vertical expresada en las dificultades de las mujeres para acceder, mantener y ascender en una calificación, así como obtener puestos de responsabilidad y de toma de decisiones que les posibilite mayor incidencia en el ámbito científico-tecnológico.

Siendo las mujeres la mitad de la población, estas desigualdades derivan en un bajo aprovechamiento de su potencial intelectual, asimismo, la evaluación más precisa de la contribución de las investigadoras se ve dificultada por la ausencia 
de información desagregada por sexo. Este hecho no permite validar adecuadamente el uso, beneficio e impacto de los recursos humanos en la ciencia y la tecnología, así como en el desarrollo socioeconómico del país. El androcentrismo en las ciencias, la segregación vertical y horizontal, la falta de visibilidad de las mujeres en la historia del saber, los estereotipos de género en las relaciones entre docentes y estudiantes, la ausencia de la perspectiva de género como eje transversal, entre otros, han sido identificados como parte del orden de poder de género que caracteriza estos espacios.

Dicho orden se produce y reproduce en lo simbólico, en los significados y sentidos, en las creencias, las tradiciones, las exigencias, habita las subjetividades sociales $e$ individuales atravesando las prácticas, los espacios, las leyes, la arquitectura, las normativas, todo.

En el caso particular de la ciencia es importante significar que el orden de poder de género se manifiesta, principalmente, en la hegemonía de un paradigma epistémico, positivista y la deslegitimación y exclusión de otros paradigmas y alternativas de producción de conocimientos. El androcentrismo en la ciencia, además de mostrarse en lo epistémico, se observa también en el clima organizacional de las instituciones científicas, en los liderazgos y sus estilos protagonizados predominantemente por hombres, en los sistemas de evaluación y reconocimiento del quehacer científico, entre otros.

A su vez, el tema de género e igualdad en la investigación científica impulsa acciones de transformación como son los diseños e implementación de políticas públicas que han tenido su expresión en México como analizaremos más adelante.

Ello permite definir un nuevo modelo para eliminar las relaciones de poder que subordinan a las mujeres y "lo femenino". Supone la toma de conciencia de la situación de las mujeres dentro de su campo de acción, la adopción de medidas que promuevan el ingreso a la ciencia y la tecnología y el fortalecimiento de las que ya se desempeñan en estos campos. Asimismo, estimula la integración de las perspectivas de las 
mujeres, sus modos de conocimiento y actuación en la construcción de paradigmas científicos y tecnológicos inclusivos, enriquecidos por la diversidad de enfoques y comprometidos con el logro de una real articulación social.

Hablamos de un asunto de equidad social, de desarrollo humano y de optimización y aprovechamiento de recursos económicos y sociales que resulta urgente en el empeño por acelerar y potenciar el desarrollo científico, económico y social que se requiere actualmente.

El presente texto se propone visibilizar este hecho y su significación, así como alentar la revisión y reflexión de políticas en ciencia y tecnología con perspectiva de género, que garanticen el camino de la transformación.

\section{¿Se entrelazan ciencia, tecnología y género?}

La limitación en el camino hacia el saber, acceder a su certificación, el ascenso y el consiguiente poder que ello supone, ha sido una de las prohibiciones más fuertes que la cultura patriarcal ha impuesto a las mujeres, lo cual comienza a revertirse a finales del siglo XX.

Hace aproximadamente tres décadas, llegan masivamente las mujeres a los espacios científicos, lo cual ha sido determinante para el logro de su autonomía y el empoderamiento, para resignificar su papel en la sociedad y a la sociedad en sí misma, así como para criticar y transformar el conocimiento.

Sin embargo, una marca de esta época es la coexistencia de espacios de innovación y reproducción del orden de poder de género. En medio de esfuerzos deconstructivos, se mantienen supuestos que sostienen el discurso y las prácticas educativas tradicionales y una única noción de "lo científico".

La desigualdad y estas relaciones de poder prevalecen, condicionando una socialización diferente, sexista, discriminatoria y excluyente de acuerdo con la condición sexuada, en detrimento del desarrollo de las mujeres y de la ciencia en general (Fernández, 2015). 
Las mujeres se encuentran aún en una relación de subordinación que se expresa de forma explícita e implícita y siguen estando excluidas de las ciencias y tecnologías más prestigiosas y de los niveles más altos del sistema, en especial de los puestos de decisión (Pérez Sedeño, 2001:46; Blazquez; Bustos, 2013).

Por su parte, Diana Maffía (2007b) afirma que el científico o la científica están determinados socioculturalmente. Una de estas determinaciones es el género, los roles asignados y las relaciones humanas condicionadas en virtud de ello. Entonces, hay que evidenciar cómo el producto del trabajo científico, supuestamente "neutral", posee un sesgo sexista.

El sesgo de género puede aparecer como subestimación de un sexo, históricamente el femenino (sexismo) y como punto de vista parcial "masculino" que hace de la experiencia del varón la medida de todas las cosas (androcentrismo). Ello ha motivado, desde hace algunas décadas, el análisis del entretejido entre género y ciencia.

Cuando se habla de estudios en ciencia, tecnología y género desde el pensamiento feminista, se está haciendo referencia al examen de la división sexual del trabajo en las ciencias y la consiguiente organización genérica de las comunidades científicas, así como de la propia ciencia que ha estimulado la desigualdad, impidiendo el acceso a la educación, ocultando o devaluando las obras escritas por mujeres y promoviendo una educación sexista presente hasta nuestros días (Pérez Sedeño, 2000).

Dichos estudios, aún en su diversidad, se oponen al sexismo y androcentrismo reflejados en la práctica científica y denuncian el dualismo dicotómico que vemos en la contraposición cantidadcalidad en el ámbito del pensamiento científico y que sostiene la ya mencionada "neutralidad" de las ciencias.

Desde este análisis complejo, podemos comprender mejor cómo se configuran: la distribución del poder y de los recursos; las decisiones de proyectos y su financiamiento; lo que se puede investigar y lo que no; el planteamiento de problemas científicos, 
hipótesis, diseños metodológicos, técnicas e instrumentos y las interpretaciones que se realizan de los datos obtenidos.

Los discursos, estereotipos y mitos sociales (científicos y de género) ordenan y legitiman, desde un orden de poder, a los actores y a los espacios sociales y subjetivos Definitivamente, tanto los valores patriarcales como los paradigmas "legítimamente científicos" se han imbricado.

Las sociedades patriarcales - prevalecientes en nuestros días - se organizan desde una prescripción de valores y normas identificables con "...una construcción simbólica que integra los atributos asignados a las personas a partir de su sexo" (Fernández, 2005:97).

Dicha construcción simbólica, que llamamos género, es "la categoría correspondiente al orden sociocultural configurado sobre la base de la sexualidad: la sexualidad a su vez definida y significada históricamente por el orden genérico" (Lagarde, 1997:11).

La diferencia sexual resignificada se expresa en un orden binario: masculino-femenino, dos modos de vida, de atributos eróticos, económicos, socioculturales, psicológicos, políticos, dos modos antagónicos de sentir y de existir.

La noción hegemónica de "lo masculino" se asocia a la fuerza, inteligencia, valentía, responsabilidad social, creatividad, racionalidad, mientras que "lo femenino" es asociado al cuidado, la maternidad, sensibilidad, dulzura, afectividad, excelencia en las relaciones interpersonales.

La división entre "lo masculino" y "lo femenino" responde también a una lógica jerárquica, es por esta razón que fundamenta la desigualdad. Quienes ostentan los atributos "superiores", los "masculinos", asignados fundamentalmente a los varones, pueden obtener con mayor facilidad posiciones de poder, autoridad y bienes en comparación con el resto (Burin, 2007). Así, las construcciones culturales de género, apoyadas en las diferencias, establecen una dicotómica jerarquización que acentúa la supremacía de lo masculino como valor y convierte lo diferente en 
desigual y devaluado. Es éste el punto cardinal del asunto: las relaciones de poder de género.

El pensamiento feminista se propone denunciar al patriarcado como sistema de jerarquización en función del sexo o como "sexualización del poder" (Hierro, 2003).

La igualdad implica igual capacidad de derechos y a lo que se opone no es a la diferencia, se opone a la desigualdad. La desigualdad también es una relación política, es la relación política de no tener el mismo acceso a los derechos (Maffía, 2007a:17).

La desigualdad es un primer elemento del mecanismo interno de las relaciones de poder entre mujeres y hombres y genera discriminación cuando se le da mayor valor a lo que hacen los hombres por sobre lo que hacen las mujeres.

Las desigualdades de género se expresan en formas diversas de discriminación en la sociedad, así como en la falta de reconocimiento del "cuidado" como generador económico que en su mayor parte es responsabilidad de las mujeres.

De esta forma, se construye una noción de "espacio público", remunerado, comprometido con el desarrollo de la sociedad, la economía, la política y la ciencia, como "masculino" y el espacio privado, no remunerado, familiar y del cuidado, como "femenino". La economía del cuidado sigue recayendo en las mujeres y continúa prevaleciendo como indicador de "feminidad".

Este hecho significa una doble jornada laboral para las mujeres en la que su trabajo extra doméstico remunerado se suma al no remunerado dentro del hogar lo cual constituye una desventaja con respecto a los hombres, quienes han permanecido predominantemente en la zona pública, productiva como definición de "masculinidad".

1 Economía del cuidado se refiere a aquellos aspectos que "son imprescindibles para la supervivencia de los miembros de la sociedad" (Rodríguez, 2005:1-2 apud Olvera Castillo, 2015) como bienes, actividades, servicios, relaciones o valores. 


\section{Mujeres en la ciencia: tensiones y dilemas}

A pesar de las transformaciones que han permitido el acceso de las mujeres a la educación y al empleo remunerado, la distribución de roles en el hogar sigue siendo sexista.

¿Podrían estos entretejidos explicar el hecho de que durante milenios se privara a las mujeres del acceso a la educación, a la ciencia y al desempeño público? ¿Podrían explicar también el hecho de que las mal llamadas "ciencias duras" sigan siendo predominantemente desempeñadas por hombres? ¿De que los cargos de dirección sigan siendo en su mayoría ejercidos por hombres? ¿De que las mujeres accedan y permanezcan en menor medida en los sistemas de reconocimiento y evaluación académica a diferencia de los hombres?

Según la Encuesta Nacional de Ocupación y Empleo en 2012 (ENOE) la tasa de participación de las mujeres en el trabajo doméstico fue de un $96 \%$ y la de los hombres de $60.9 \%$. Casi el $100 \%$ de las mujeres mexicanas encuestadas participa en labores domésticas no remuneradas (Olvera, 2015:4).

Es así que las mujeres investigadoras tienen menor oportunidad que sus pares investigadores para realizar las actividades que les exige el sistema ya que por lo general deben asumir labores de cuidado que los hombres realizan en menor proporción. Según la Encuesta Nacional sobre Uso del Tiempo (INEGI, 2003) en México el trabajo doméstico representó $21.6 \%$ del PIB nacional de ese año.

Lo anterior permite apreciar cómo se producen $\mathrm{y}$ reproducen en lo simbólico relaciones de poder, así como un lenguaje social, modelos y mecanismos para la interiorización que cristalizan en las subculturas de género, en las creencias, las tradiciones, anidando en las subjetividades sociales $e$ individuales.

La tensión entre la sobre-exigencia de los espacios académicos y la conservación de asignaciones tradicionales de género tienen una incidencia en la composición presencial del 
profesorado y del alumnado, que aunque ha ido incorporando a más mujeres, todavía no alcanza la equidad por nivel o por área del conocimiento. Por ejemplo, las cifras muestran que hasta los 14 años las mujeres son mayoría en las instituciones escolares del país, pero a partir de esta etapa se invierte su representación y del porcentaje de la población de 20 a 29 años que llega al nivel de educación superior en México (que es solo de una persona por cada 10) el $19 \%$ son hombres y el $16 \%$ son mujeres (INEGI, 2015). Partiendo de esos porcentajes, la matrícula de mujeres y hombres a nivel licenciatura es prácticamente de 50 y $50 \%$. Sin embargo, aun y cuando se reconoce una reducción de las brechas debido a un creciente ingreso de ellas al sistema educativo, persisten desventajas para la población femenina en la continuidad de sus estudios y por áreas del conocimiento.

Un fenómeno interesante es que algunas áreas del conocimiento que a principios de la década de los ochenta ocupaban un porcentaje mayor de hombres, actualmente tienen una matrícula mayor de mujeres. Tales son los casos de ciencias de la salud, así como ciencias administrativas, y en carreras específicas como veterinaria e ingeniería textil, por citar algunas. Sin embargo, siguen existiendo considerables disparidades en ciertas áreas del conocimiento como las ingenierías, ciencias exactas y agronomía (Quintana, 2016).

En el caso de la Universidad Nacional Autónoma de México (UNAM), una de las más grandes e importantes de Iberoamérica, las mujeres representan $53 \%$ de la población total de estudiantes de licenciatura y $50 \%$ en posgrado, sin embargo, cuando se analiza la matrícula por áreas del conocimiento, el comportamiento es distinto, ya que se observan carreras con un número muy bajo de mujeres como ingeniería y física, y otras con una población femenina mayor como pedagogía, enfermería o psicología.

En cuanto al total del personal académico de la UNAM, se observa que asciende a 36,172 personas, $43 \%$ son mujeres y $57 \%$ son hombres y esta diferencia se hace mayor al desagregar por 
categoría ya que los hombres tienen un mayor porcentaje en las más altas (Blazquez; Bustos, 2013; Blazquez, 2014).

Asimismo, los empleadores en ocasiones asumen y asignan roles desde el sexismo tradicional y automáticamente las mujeres quedan excluidas de trabajos no compatibles en tiempo $y$ dedicación con las labores domésticas que "deben protagonizar" (Virgilí, 2012). Mientras, el diseño de la vida pública sigue siendo androcéntrico: dedicación extrema, largas jornadas de trabajo y actividades en horarios extendidos, como si fuera éste el único espacio de vida más privilegiado.

Estos datos permiten afirmar que se continúan perpetuando estereotipos de género como expresión de una cultura androcéntrica. La conciliación del espacio público y privado, el éxito en dichos espacios, la entrega y el tiempo que exige la superación profesional, la doble jornada laboral, el sobreuso del tiempo en las mujeres así como las nuevas concepciones en torno a las relaciones de pareja, de familia, de maternidad y paternidad, son cuestiones que impactan de modo significativo la vida de quienes laboran en la academia acompañado de insuficiente reflexión consciente en cuanto a la condición de género, a sí mismos, a sí mismas y a su realidad.

Se sigue privilegiando una noción positivista de las ciencias. Las mujeres habitan este espacio que se conserva tal cual sin una transformación personal y social. La causa está en la propia sociedad donde anida la cultura patriarcal. Por eso, para Sandra Harding (1996), el relativo fracaso de los esfuerzos por integrar a niñas y mujeres en la ciencia y la tecnología conduce a estimular una reflexión que transite del análisis de las mujeres en las ciencias al análisis de cuáles serían los supuestos cambios en la noción de ciencia, sus contenidos, métodos y prácticas desde una mayor presencia de las mujeres y del pensamiento feminista.

Una perspectiva de género ayuda a develar aquellas prácticas de inequidad que han sido justificadas y racionalizadas en beneficio del ser y quehacer de los hombres. Reveladas éstas desigualdades es posible intervenir en la realidad para cambiar dichas relacionas jerárquicas hacia nuevas formas democratizadas 
de interactuar, cuya base ética es la igualdad entre seres humanos. Dicha perspectiva permite formular recomendaciones que favorezcan el ingreso, permanencia y ascenso de las mujeres académicas y contribuir a su propio bienestar, el de las familias y la sociedad.

En este sentido resultan de especial utilidad tres conceptos: división sexual del trabajo, doble jornada laboral y conciliación con corresponsabilidad.

La división sexual del trabajo es "el reparto social de tareas en función del sexo, según el cual históricamente se asigna a las mujeres el trabajo reproductivo y a los hombres el trabajo productivo". Los hombres tienen la responsabilidad de proveer ingresos familiares y las mujeres la obligación del cuidado en el mundo doméstico incluyendo la crianza de hijas e hijos (DT, 2012: 21 \& UNFPA, 2005:128 apud Olvera Castillo, 2015).

Doble jornada, es la "suma de la jornada laboral remunerada y la jornada posterior dedicada principalmente en las tareas del hogar y cuidados de personas dependientes" (DT, 2012:21 apud Olvera Castillo, 2015). La doble jornada es uno de los principales factores de discriminación salarial y laboral. En el caso de las mujeres académicas esto limita la posibilidad de alcanzar igual ritmo de ascenso científico y por tanto igual nivel de estímulos económicos que los investigadores.

Todo esto repercute en el acceso a los sistemas de reconocimiento y estímulos económicos como el Sistema Nacional de Investigadores $(\mathrm{SNI})^{2}$ que requieren del título doctoral (Blazquez, 2014; Mendieta, 2015). Algunas mujeres deciden retrasar o denegar sus planes de formar una familia para poder cumplir con este requisito. Otras, deciden tener hijos y una vez avanzada la independencia de estos implementan sus intenciones de estudiar el doctorado y posteriormente pertenecer al SNI. En esta disyuntiva, la mayor parte de ellas no llega. Otras se ven atrapadas en los cortes de edades que sitúa la academia en el acceso a

\footnotetext{
2 El Sistema Nacional de Investigadores se creó en 1984 para reconocer y
} estimular la labor de investigación en México. 
espacios doctorales y de superación continua. Ello se entrelaza con diversas dimensiones de exclusión: la condición sexuada y las asignaciones de género, el derecho y decisión del maternazgo, la edad, la condición étnica, socioeconómica, procedencia geográfica, entre otras.

El aplazamiento en la edad vuelve a encontrarse con las tareas de cuidado de personas dependientes en la adultez mayor, dado el aumento de la esperanza de vida en las sociedades contemporáneas lo cual sigue siendo un asunto de las mujeres. Una estrategia relativamente frecuente es delegar los roles tradicionalmente asignados en otras mujeres familiares $\mathrm{o}$ remuneradas.

Se trata de un dilema sostenido en la subjetividad social desde la asunción de lo asignado y las propias exigencias socioeconómicas en los contextos específicos. Dicho dilema es vivido de modo contradictorio, tenso y conflictivo por muchas mujeres con consecuencias para la vida personal, familiar y social (Blazquez, 2014; Mendieta, 2014).

Esta subjetivación de la cultura patriarcal se enfrenta con una cultura crítica que permite cambiar e innovar en la asunción de lo asignado pero que emerge lentamente. Los hombres, como tendencia, no tienen este dilema tenso entre vida académica y vida personal-familiar. Por lo general cuentan con alguien que se encarga de labores domésticas, de crianza y de cuidado.

Se han realizado varias propuestas para aliviar la tensión entre los ámbitos familiar, laboral y personal. Sin embargo, dichas propuestas no siempre favorecen las necesidades de las mujeres ya que algunas llegan a perpetuar los roles y estereotipos de género relacionados con lo "masculino" y lo "femenino" al ser políticas de familia que no concuerda con la diversidad de familias actuales (Mendieta, 2014:58-60).

Aparecen propuestas para que se aprueben los permisos de paternidad remunerados, que consisten en la posibilidad de un período de tiempo de ausencia laboral para la figura paterna con disfrute de salario, que pueden ser acciones de cambio pero que a la vez reproducen la división sexual de tareas en el ámbito 
doméstico. Sin embargo, se podría pensar en alternancias o conjunción de cuidados parentales durante los períodos de mayor dependencia y en continuidad para la crianza y educación de la descendencia.

Para que se pueda llegar a una configuración más igualitaria de éstas esferas es indispensable tomar en cuenta la conciliación con corresponsabilidad entre el terreno laboral, familiar y social. No se trata de conciliar para que las mujeres puedan "cumplir" con todos los roles y perpetuar el modelo de la super woman sino de pensar y lograr dinámicas más equitativas e igualitarias entre todas las personas.

La corresponsabilidad supone una participación equilibrada de cada integrante de la familia en las tareas y responsabilidades de la vida familiar y el cuidado de personas dependientes de acuerdo a sus capacidades.

El PNUD (2010:18) incluye al Estado y al mercado para que asuman una redistribución de responsabilidades que se han dejado solamente a las familias, especialmente a las mujeres, puesto que la "conciliación con corresponsabilidad social" que facilite a las/los integrantes de la familia combinar el trabajo remunerado con el de cuidado es indispensable para alcanzar el desarrollo humano y la igualdad de género.

La carencia en las políticas públicas no estimula la participación de los hombres en el trabajo reproductivo y dificultan la plena integración de las mujeres al mercado laboral. El tema queda relegado a estrategias y decisiones personales de las mujeres para satisfacer sus necesidades de cuidados y sus deseos de autonomía económica, profesional, social, subjetiva.

\section{Políticas públicas}

Las Políticas Públicas pueden entenderse como un espacio de "pacto" entre Estado y sociedad con el objetivo de resolver y dar respuestas a la multiplicidad de necesidades, intereses y preferencias de grupos y personas que integran una sociedad de 
forma tal que las soluciones encontradas permitan que personas y grupos coexistan en sus diferencias.

Puede concebirse también como un proceso de solución de problemas públicos (Aguilar, 2010) con varios momentos con un orden lógico que definen varias fases interdependientes.

Para concebir una política pública, es imprescindible partir de las necesidades de los grupos sociales a quienes van a ir dirigidas y así, poder llevar a cabo proyectos reales, viables y sustentables, para lo cual se necesita del trabajo interdisciplinario que contribuya al análisis, diseño, planeación, evaluación de acciones gubernamentales intencionales en los ámbitos de la educación, salud, seguridad pública, infraestructura, comunicaciones, energía, agricultura, entre otras.

Los tres componentes principales de cualquier política son: los principios que la orientan; los instrumentos mediante los cuales se ejecuta la regulación, financiamiento, y mecanismos de prestación de las políticas y los servicios o acciones principales que deberían llevarse a cabo de acuerdo a los principios propuestos.

Incluye un diagnóstico de algún aspecto de la vida social que se considera polémico llegando a ser un problema público que necesita cambios. Esta etapa de problematización implica recabar información confiable, resultados de investigaciones teóricas, historias de éxito, aplicación de tecnologías, conocimientos previos para la definición de los objetivos y las acciones hacia la solución del problema.

Otro momento es la previsión del impacto que podría tener la Política Pública al momento de su implementación, reacciones, grupos en contra y a favor, etc. Para intuir los posibles escenarios, se requiere una gran visión política, por lo cual se sugiere el asesoramiento de personas que pudieran contar con esta poderosa herramienta. La representación de un pronóstico al proponer una solución al problema precisa lo que se debe hacer y fundamenta las razones para implicarse en la acción, proporciona un mayor índice de seguridad, así como el aumento de las probabilidades de éxito de la política que se quiere impulsar. Luego, el establecimiento de objetivos del futuro deseado en un ámbito 
determinado y que serán resueltos en el transcurso de la Política Pública, pueden ir acompañados del tiempo en el cual se quieran resultados a fin de ir revisando paulatinamente si se van cumpliendo. Se definen alternativas de política para intervenir de la mejor manera, además se delibera sobre la factibilidad de los objetivos. En esta etapa se diseña el cómo se pondrá en práctica lo acordado. La implementación se lleva a cabo una vez que ya se tienen todos los pasos anteriores.

Esto será evaluado junto con la calidad de los productos y quienes operaron el programa, con la finalidad de mejorar o corregir la política y así volver a empezar el ciclo. Este paso permite realmente ver desde el comienzo algunas fallas o las que se vayan dando en el transcurso de la implementación, así como los mecanismos precisos para la ejecución, los instrumentos para evaluar las acciones, resultados e impactos asociados y la modificación para mejorar la política y así volver a empezar el ciclo (Villarreal, 2013 apud Olvera Castillo, 2015).

Generalmente se tienen los siguientes instrumentos para plantear e impulsar las Políticas Públicas:

1. Las normas jurídicas a través de las cuales los poderes públicos autorizan y establecen las actividades que constituyen las políticas, y limitan la discrecionalidad en el actuar de los que la elaboran y ejecutan.

2. Los servicios de personal, ya que al elaborar las políticas se requiere infraestructura humana, organizativa y de material.

3. Los recursos materiales, en especial los financieros que suelen ser más restringidos.

4. La persuasión, pues los ciudadanos consideran al gobierno como legítima expresión de la interpretación de los intereses generales de la sociedad y el gobierno debe velar por los intereses sociales.

\section{Políticas de ciencia, tecnología y género: antecedentes}

Desde la década de los años setenta en el contexto internacional se ha elaborado un cuerpo legislativo de gran 
importancia para el avance en la igualdad de género que es de obligatorio cumplimiento para los Estados que los han suscrito y ratificado. Es posible presentar un recorrido sintético de tales acciones internacionales al abordar los temas de género, educación superior, ciencia y tecnología:

En la Declaración y Plataforma de Acción de la cuarta Conferencia Mundial de la Mujer de las Naciones Unidas, realizada en Beijing en septiembre de 1995 se acordó:

- Eliminar disparidades de género en el acceso a la enseñanza; igual acceso de las mujeres al desarrollo profesional, la capacitación y las becas, con medidas de promoción activa.

- Proporcionar a las mujeres y niñas información sobre formación profesional, programas de capacitación en ciencia y tecnología y programas de educación permanente.

- Diversificar la formación profesional y técnica; aumentar el acceso y retención de niñas y mujeres en los campos de las ciencias, las matemáticas, la ingeniería y la tecnología.

- Aumentar la proporción de mujeres en la elaboración de políticas y toma de decisiones en materia de educación en todos los niveles de la enseñanza y en todas las disciplinas académicas, principalmente las que están dominadas por hombres.

- Aumentar el número de mujeres en puestos de dirección en las profesiones de la salud y entre los investigadores y científicos para alcanzar la igualdad.

- Fomentar políticas y medidas de género a fin de crear condiciones para que las mujeres puedan participar en igualdad con los hombres en los campos técnico, administrativo y empresarial.

- Reconocer y alentar la contribución de las investigaciones de las científicas y las tecnólogas.

- Apoyar y realizar investigaciones de género en todos los niveles de la enseñanza en las instituciones académicas, y aplicarlos en la elaboración de programas, libros de texto, material didáctico y formación de personal docente.

- Estimular y reconocer las redes de comunicación de mujeres, 
entre ellas las redes electrónicas para la difusión de información y el intercambio de ideas.

- Aumentar la proporción de mujeres que participan como dirigentes, planificadoras, administradoras, científicas y asesoras técnicas en el diseño, desarrollo y ejecución de políticas y programas para los distintos campos de la ciencia.

- Elaborar bases de datos y sistemas de información y llevar a cabo investigaciones, metodologías y análisis de políticas y dinámicas en que se tenga en cuenta el género, con la colaboración de las instituciones académicas y de las investigadoras.

- Elaborar y aprobar programas de estudios, material didáctico y libros de texto que mejoren el concepto de sí misma de las niñas, sus vidas y sus oportunidades de trabajo, especialmente en áreas en que las mujeres han estado menos representadas, como las matemáticas, la ciencia y la tecnología.

En la Declaración de la Conferencia Mundial de Ciencia. "Ciencia y el uso del Conocimiento Científico", realizada en Budapest, Hungría en junio de 1999, se afirmó que, para asegurar un desarrollo sostenido, es fundamental la educación científica en todos los niveles y sin discriminación de ningún tipo. En este sentido se puntualizó que:

- La práctica de la investigación científica y el conocimiento científico deben lograr el bienestar de la humanidad, considerando la igualdad entre hombres y mujeres; deben respetar la dignidad de los seres humanos y de sus derechos fundamentales, tomando en cuenta a las generaciones futuras.

- Las dificultades de las mujeres para entrar y terminar la carrera científica, así como lograr el acceso a puestos de decisión, debe atenderse mediante mecanismos institucionales y con medidas que lleven a esas metas.

- La igualdad en el acceso a la ciencia es un requerimiento social y ético para el desarrollo humano, y es una necesidad para alcanzar el potencial de las comunidades científicas y 
orientar el progreso científico hacia el logro de las necesidades humanas.

En la Reunión Hemisférica de Expertos en Género, Ciencia y Tecnología de la Oficina de Ciencia y Tecnología de la Organización de los Estados Americanos y la Comisión Interamericana de la Mujer de las Naciones Unidas, realizada en Washington, EU, en agosto de 2004, se planteó:

- Incluir la perspectiva de género en las políticas y programas de ciencia y tecnología.

- Apoyo con presupuestos, para que las mujeres tengan igual representación y avance en ciencia y tecnología, así como en foros y cuerpos de toma de decisiones.

- Creación, adquisición, utilización y diseminación del conocimiento con la participación de las mujeres. Integrar perspectivas de género en ciencias exactas, naturales y sociales, tecnología, ingeniería e innovación y generar, recolectar y diseminar el conocimiento para fundamentar políticas y tomar decisiones.

- Mejorar la calidad de la educación en ciencia y tecnología en todos los niveles, con la eliminación de prejuicios de género y la promoción de creatividad y pensamiento crítico.

- Incorporar mujeres y niñas a la ciencia y la tecnología, y capacitación para la promoción, retención y avance de las mujeres en la fuerza de trabajo activa de ciencia y tecnología.

- Desarrollar y poner en práctica políticas nacionales y regionales

sobre género y desarrollo social, ciencia y tecnología, en conjunción con los ministerios relacionados, así como con instituciones de género nacionales.

- Construir la sociedad de conocimiento mediante equidad e igualdad de género. Acceso igual de las mujeres a la utilización y beneficios de las nuevas tecnologías

- Promoción de género. Incluir educación y capacitación, oportunidades de empleo y desarrollo profesional, así como mayor investigación en género y ciencia. 
Los Antecedentes de Política Científica y Género poseen también su expresión en el contexto regional y nacional:

La incorporación de las mujeres en las universidades en el siglo XX ha permitido el crecimiento y consolidación de la participación de académicas y científicas feministas, quienes han abordado temas ignorados o dejados de lado que poco a poco han influido en las políticas públicas.

Se pueden citar algunos avances e iniciativas tanto en las agendas nacionales y regionales de investigación y acción en ciencia y tecnología, en los diversos mecanismos de interacción entre el gobierno y las organizaciones de mujeres a favor de la equidad de género y la no discriminación, así como en la definición de políticas y programas en el tema:

- En 1974 en México se modificó el Artículo $4^{\circ}$ de la Constitución Política, que estableció la igualdad jurídica entre la mujer y el hombre.

- En noviembre de 2000, se realizó el Taller sobre Indicadores de Impacto Social de la Ciencia y Tecnología organizado por la Red Iberoamericana de Indicadores de Ciencia y Tecnología (RICYT) en Argentina (Argenti, 2000).

- En 2001 se celebró el Taller de Indicadores de Género, Ciencia y Tecnología en Uruguay.

- En 2002 se publicó en México el Programa Nacional para la Igualdad de Oportunidades y la No Discriminación contra las Mujeres.

- En 2004 se llevó a cabo la Reunión Hemisférica de Expertos en Género, Ciencia y Tecnología, cuyas recomendaciones fueron aprobadas por los Ministerios de Ciencia y Tecnología, y se incluyeron en las iniciativas hemisféricas de la Declaración y Plan de Acción de Lima, Perú. Se prepararon dos documentos sobre el tema: uno de carácter regional (Bonder, 2004) y otro internacional (Huyer, 2004).

- En ese mismo año, 2004, se realizó en nuestro país el V Congreso Iberoamericano de Ciencia, Tecnología y Género, 
como parte de las actividades de la Red Iberoamericana de Género, Ciencia y Tecnología (Blazquez; Flores, 2005).

- En 2005 y 2006 se celebraron la I y II Conferencia Latinoamericana de Mujeres en las Ciencias Exactas y de la Vida, en Brasil y en México respectivamente (Meza Montes cols., 2009).

- En cuanto a las políticas del gobierno en materia científica, puede decirse que ha habido una influencia gracias a las aportaciones y el intenso activismo social y académico que han realizado los diversos grupos de académicas y científicas del país desde hace 35 años, a fin de lograr la equidad de género en el desarrollo científico de México, como ocurrió con el análisis realizado en el II Simposio Ciclo Mujer Ciencia, UNAM, en la mesa redonda titulada: La Visión de las Científicas sobre la propuesta: "Hacia una Política de Estado en Ciencia, Tecnología e Innovación en México del Foro Consultivo Científico y Tecnológico", realizada en 2007 en el marco del II Ciclo Mujer y Ciencia UNAM (Pérez Armendáriz, 2010).

Sin embargo, con todos estos esfuerzos, las propuestas del Foro Consultivo Científico y Tecnológico (FCCyT) y de la Ley de Ciencia Tecnología e Innovación (CTI), hasta la primera década del año 2000, no tenían recomendaciones para la inclusión de la equidad e igualdad de género en la participación de las mujeres en el desarrollo científico. Tampoco contemplaban el acuerdo interamericano previamente aprobado en la Primera Reunión de Ministros y principales representantes en Ciencia y Tecnología, convocada por la OEA y realizada en Lima, Perú, en el 2004.

La no inclusión de indicadores de género hasta fechas recientes ha limitado el diagnóstico y las líneas de acción para el diseño y desarrollo de políticas en las instituciones en las que se realiza y dirige el desarrollo de actividades científicas y tecnológicas que estimulen cada vez más la participación de las mujeres en estas actividades en condiciones de igualdad.

Es por ello muy importante ilustrar los recientes avances: 
- En 2013, el Consejo Nacional de Ciencia y Tecnología $\left(\right.$ CONACYT) ${ }^{3}$ formalizó acciones a favor de la igualdad de género en las reformas a los artículos de la Ley de Ciencia y Tecnología:

\section{Fracción VIII}

"Promover la inclusión de la perspectiva de género con una visión transversal en la ciencia, la tecnología y la innovación, así como una participación equitativa de mujeres y hombres en todos los ámbitos del Sistema Nacional de Ciencia, Tecnología e Innovación".

Fracción $\mathrm{V}$

12: “...incentivar la participación equilibrada y sin discriminación entre mujeres y hombres y el desarrollo de las nuevas generaciones de investigadores y tecnólogos"

14: "En la medida de lo posible, el sistema deberá incluir información de manera diferenciada entre mujeres y hombres a fin de que se pueda medir el impacto y la incidencia de las políticas y programas en materia de desarrollo científico, tecnológico e innovación."

42: "El gobierno federal apoyará la investigación científica y tecnológica que contribuya significativamente a desarrollar un sistema de educación, formación y consolidación de recursos humanos de alta calidad en igualdad de oportunidades y acceso entre mujeres y hombres" (GM, 2014:3-26 apud Olvera Castillo, 2015)

También se puede destacar el Programa Nacional para la Igualdad entre Mujeres y Hombres (PROIGUALDAD 2013-2018 apud Olvera Castillo, 2015) en donde se propone:

3 La forma institucional en México para la definición de la ciencia, la tecnología y la innovación es el Consejo Nacional de Ciencia y Tecnología (CONACYT), organismo que articula las políticas públicas del Gobierno Federal para promover la investigación y formación de investigadores e investigadoras. 
“...implementar programas para la corresponsabilidad entre la vida laboral, familiar y personal, con participación de empresas, gobierno y trabajadoras/es, incrementar la infraestructura para apoyar las tareas del cuidado" (GM, 2013:3 apud Olvera Castillo, 2015) haciendo énfasis en el cumplimiento de la Ley Federal del Trabajo.

Con esos antecedentes sumados a la coincidencia afortunada de dos mujeres muy destacadas en cargos de decisión en CONACYT y en el Foro Consultivo Científico y Tecnológico (FCCyT) - las doctoras Julia Tagüeña y Gabriela Dutrenit respectivamente -, ha sido posible la incorporación de la perspectiva de género en esas instituciones:

- En 2013 se producen acercamientos entre el Foro Consultivo Científico y Tecnológico en colaboración con diversas instituciones y grupos de académicas al introducir una visión de la ciencia, la tecnología y la innovación con perspectiva de género y diseño de políticas públicas (FCCYT, 2013).

- El Reglamento del SNI incluyó en el Artículo 13: "Las comisiones dictaminadoras se integrarán por catorce miembros; en ellas se procurará equilibrio y paridad entre disciplinas, instituciones, sexo y regiones" (Reglamento SNI, 2013). Ello es un paso, pero no garantiza linealmente la equidad, si quienes integran dichas comisiones no son académicas/os flexibles, sin estereotipos y con una cosmovisión inclusiva, proclive a la igualdad y la no discriminación.

- Adicionalmente, el Artículo 62 de ese mismo Reglamento indica: "A las investigadoras cuyo embarazo ocurra durante el periodo de vigencia de su distinción, se les otorgará un año de extensión, mediante solicitud expresa de la interesada..." (Conacyt, 2013 apud Olvera Castillo, 2015).

Todo este trabajo colectivo y las propuestas de diversas organizaciones de académicas y científicas han logrado que en los últimos tres años se empiece a hablar de una política de ciencia y 
tecnología con perspectiva de género. Como ejemplos se puede citar el proyecto "miseal" coordinado por la Universidad Estadual de Campinas en Brasil, en conjunto con el Instituto de Estudios Latinoamericanos de la Freie Universität Berlín, en el que participan 12 universidades latinoamericanas y 4 europeas que proponen medidas para la inclusión social y la equidad en instituciones de educación superior en América Latina, (http://www.miseal.net/), así como las propuestas derivadas de diversas reflexiones en foros de discusión organizados por Grupos y Redes de Académicas de México e Iberoamérica (Pérez Armendariz, 2015; www.redmexciteg).

Políticas de ciencia, tecnología y género: reflexiones y propuestas actuales.

Las nuevas ideas dirigidas a alcanzar la igualdad se centran en estrategias que promueven cambios profundos en el orden de poder y los roles de género. Las mismas se orientan a modificar los estereotipos de género referidos a las funciones y tareas dentro de las familias y fuera de ellas, en los ámbitos productivos, comunitarios y personales.

El cambio hacia una mayor igualdad en el ámbito público y en el ámbito privado tendría que cuestionar a quienes dirigen las acciones y a quién se considera el grupo problemático. Hacen falta acciones que pretendan verdaderos cambios a largo plazo y que reconsideren concepciones de la vida familiar y laboral.

Las decisiones en materia científica forman parte de las políticas de cada gobierno siendo esencial la incorporación de la perspectiva de género en las instituciones en las que se realizan estas actividades.

Ello contribuye a la solución de las necesidades nacionales, a la eliminación de las persistentes desigualdades entre hombres y mujeres, así como a disminuir los costos personales, familiares y sociales derivados de la insuficiente corresponsabilidad en la vida privada, familiar y de políticas tendientes a avanzar en equidad en el ámbito privado y público. 
Actualmente el sistema de ciencia y tecnología apuesta por la ventaja competitiva que representa la utilización más eficaz del capital humano cuando en el mismo están representadas las mujeres. De este modo, al reconocer la importancia de las condicionantes de género, se incluyen y reconocen de manera ampliada diversos talentos, conocimientos y experiencias de las mujeres. Ello crea nuevas oportunidades económicas y científicas al diversificar los puntos de vista y proporcionar un conocimiento más profundo de la sociedad.

Una mayor inclusión de las mujeres en los sistemas de investigación, como el Sistema Nacional de Investigadores, en los programas de estímulo de las instituciones de educación superior y la inclusión de la perspectiva de género en la investigación científica mejorará la calidad, objetividad y relevancia del conocimiento para la sociedad.

Finalmente, no solo se trata de incluir a las mujeres en la ciencia construida en su mayoría por hombres, se trata de reestructurar paulatinamente a las instituciones de educación superior y de investigación científica que garanticen la equidad y la igualdad para un desarrollo pleno de la sociedad.

Para conseguir esto es imprescindible una verdadera coeducación de niños y niñas que remueva las asignaciones patriarcales de género $y$ reformule en el concepto $y$ en las prácticas el "ser mujer" y el "ser hombre" así como enfocar el problema desde un reparto más equitativo de las tareas relacionadas con el cuidado.

La conciliación corresponsable de trabajo y familia tiende a enmarcarse dentro de la 'organización del trabajo' en vez de la 'organización de la intimidad', pues no es solamente un asunto privado, sino que es responsabilidad pública. Esto significa que la norma democrática debería gobernar no solamente la sociedad sino también la familia. Asimismo, otorgar un mayor reconocimiento al papel de los hombres como padres y cuidadores y cuestionar el papel principal de las mujeres como cuidadoras universales. 
Se considera que el fenómeno de la corresponsabilidad deriva de la voluntad y/o necesidad de las mujeres de entrar en el mercado del trabajo, de la necesidad de jerarquizar el trabajo productivo en la esfera pública sobre el trabajo supuestamente "reproductivo" en la esfera privada.

Contradicción entre el diagnóstico, en el que se responsabiliza a la estructura social tradicional por el problema, y el pronóstico, que se centra exclusivamente en las mujeres para que éstas 'se animen a participar' en ciencia y tecnología.

Por ello es una necesidad colocar los trabajos de cuidados y el derecho de ser cuidado/a en el centro de las políticas públicas (Martínez, 2010:5 apud Olvera Castillo, 2015).

El Programa de las Naciones Unidas para el Desarrollo realiza una propuesta que consiste en tres tipos de estrategias. Las primeras son estrategias de secuencia en las que se alternan los tiempos destinados a lo familiar y laboral para que no se empalmen. Las segundas son estrategias derivativas en las que se trasladan las tareas reproductivas a servicios públicos o privados y las terceras estrategias proponen reorganizar los roles redistributivos y promueven cambios culturales del estereotipo femenino y masculino (PNUD, 2010).

Entre las posibles recomendaciones para continuar la elaboración de una política de ciencia y tecnología con perspectiva de género (Fernández; Blazquez, 2015) ${ }^{4}$ se distinguen las siguientes:

- Incorporar en el marco jurídico de los diferentes actores e instancias gubernamentales que rigen la actividad científica, tecnológica y la educación, la obligatoriedad de presentar información desglosada por sexo que sea pública, accesible y actualizada.

${ }^{4}$ Conclusiones del Primer Coloquio Iberoamericano: Diálogo de Saberes y Políticas de Ciencia, Tecnología e Innovación con perspectiva de Género. Cuernavaca Morelos, México. 23-25 de Junio de 2015 (http://www.ifuap.buap.mx/eventos/IColoquiol2015/index.html). 
- Desarrollar y difundir Indicadores de Género en Ciencia y Tecnología.

- Continuar con la incorporación de la perspectiva de género en la Ley de Ciencia, Tecnología e Innovación y armonizarla con los reglamentos que se deriven para el sistema de ciencia y tecnología del país.

- Continuar la transversalización de la perspectiva de género en las instituciones científicas y de educación superior.

- Incorporar en los comités editoriales, asesores, dictaminadores, revisores y científicos personal experto en estudios de género que garanticen un arbitraje o evaluación fundamentada y de calidad.

- Perfeccionar el sistema de reconocimiento, evaluación y estímulo del campo de los estudios de Ciencia, Tecnología y Género.

- Garantizar la paridad presencial de mujeres y hombres en las Comisiones Dictaminadoras y Revisoras del Sistema Nacional de Investigadores.

- Incorporar personal experto en estudios de género en estas Comisiones Dictaminadoras y Revisoras.

- Promover de manera particular el ingreso y desarrollo de mujeres y hombres en áreas con infra-representación en cada caso y con ello contribuir a reducir la segregación horizontal de las ciencias.

- Estimular con becas, proyectos de investigación y plazas nuevas la participación de mujeres jóvenes en la ciencia.

- Cambiar los límites de edad en las políticas de asignación de becas, de estancias y proyectos de investigación, que tomen en cuenta los diferentes ciclos de vida y en particular el ciclo reproductivo promoviendo así una perspectiva inclusiva de género y edad.

- Promover medidas que favorezcan el cuidado de hijas, hijos y personas dependientes fomentando la equidad de género, la corresponsabilidad y la cultura del cuidado.

- Crear y operar un observatorio de género y ciencia en los medios de comunicación 
- Contemplar acciones presupuestales para fomentar y desarrollar la ciencia y la tecnología del país desde una perspectiva de género.

\section{Reflexión final}

Uno de los sucesos más innovadores de las sociedades contemporáneas, es el acceso creciente de las mujeres al saber de más alto nivel, la acreditación de ese saber y el ascenso de las mismas a puestos de toma de decisiones. Sin embargo, aún siguen existiendo barreras que les impiden participar en condiciones de igualdad en relación con los hombres.

Las nuevas propuestas dirigidas a alcanzar la igualdad se orientan a cambios profundos que reviertan el orden de poder de género y su expresión en el ámbito de la ciencia y la tecnología.

Para ello se requieren acciones que produzcan verdaderos cambios a largo plazo y que resignifiquen las concepciones de la vida familiar y laboral, que deconstruyan los estereotipos de género prevalecientes en los ámbitos productivos, comunitarios y personales, así como en la propia ciencia.

La incorporación de la perspectiva de género en las instituciones científicas y de educación superior permite comprender y develar el orden de poder de género en estos ámbitos y contribuye, con sus propuestas de cambios, a la solución de las necesidades nacionales, a la eliminación de las persistentes desigualdades entre hombres y mujeres, a disminuir los costos personales, familiares y sociales derivados de la insuficiente corresponsabilidad en la vida familiar y a avanzar en equidad social.

En el contexto mexicano persisten desigualdades $y$ polarización social de diversos órdenes que limitan, en buena medida, el avance de la igualdad en ciencia, tecnología y género. Sin embargo, se impulsan paulatinamente políticas de inclusión que indican la presencia cada vez más acentuada de visiones interseccionales. Ello es de especial importancia pues las condicionantes de género y el orden de poder que entrañan, se 
manifiestan de modo contextualizado $e$ interpenetrado con otros órdenes de poder para dar cuenta de una opresión múltiple que es necesario atender cuando se examinan, diseñan e implementan políticas públicas a favor de la igualdad en ciencia, tecnología y género.

Ello crea nuevas oportunidades económicas y científicas al diversificar los puntos de vista, potenciar y optimizar el talento científico y proporcionar un conocimiento más amplio y profundo de la sociedad.

\section{Referencias bibliográficas}

Aguilar, Luis. Política Pública. México, D.F., Siglo veintiuno, 2010.

Argenti, Gisela. Equidad de Género en Ciencia y Tecnología. Ponencia presentada en el III Taller sobre Indicadores de Impacto Social de la Ciencia y Tecnología. Red Iberoamericana de Indicadores de Ciencia y Tecnología (RICYT), Buenos Aires, Argentina, 2000.

BlazQueZ GRAF, Norma; FloreS, Javier (eds.). Ciencia, Tecnología y Género en Iberoamérica. CEIICH, UNAM, UNIFEM y Plaza y Valdés, México, 2005.

BlazQuez Graf, Norma; Bustos Romero, Olga. Saber y Poder. Testimonios de Directoras de la UNAM. CEIICH, DGAPA UNAM. México, 2013.

BLAZQUEZ GRAF, Norma (coord.) Evaluación Académica: Sesgos de Género. CEIICH, UNAM/CYTED, México, 2014.

BONDER Gloria. Equidad de Género en Ciencia y Tecnología en América Latina: Bases y Proyecciones en la Construcción de Conocimientos, Agendas e Institucionalidades. Oficina de Ciencia y Tecnología de la Organización de los Estados Americanos y la Comisión Interamericana de la Mujer de las Naciones Unidas, Washington D.C., 2004.

BuRIN, M. Precariedad laboral, masculinidad, paternidad. In: BuRIN, M.; JiMÉNEZ, L.; Meler I. (comp.) Precariedad laboral y crisis de la masculinidad. Impacto sobre las relaciones de género. Universidad de 
Ciencias Empresariales y Sociales (UCES), Buenos Aires, 2007, pp.87-120.

CUADERNOS de Trabajo de la Red Mexicana de Ciencia, Tecnología y Género. 2016 [www.redmexciteg.org].

DECLARACIÓN y Plataforma de Acción de la cuarta Conferencia Mundial de la Mujer de las Naciones Unidas. Beijing, 1995.

DEClaración de la Conferencia Mundial de Ciencia. "Ciencia y el uso del Conocimiento Científico”. Budapest, Hungría. 1999.

ENCUESTA Nacional sobre uso del Tiempo, INEGI, 2003.

FERNÁNDEZ, Lourdes; VIRGILÍ, Dalia; Alfonso, Rachel. Creando espacios, sensibilizando en género. CEIICH, UNAM, México, 2015.

FERNÁNDEZ RIUS, Lourdes. Género, ciencia y educación en valores. In: FERNÁNDEZ RIUS, Lourdes (coord.). Género, valores y sociedad. Una alternativa desde Iberoamérica. España, Organización de Estados Iberoamericanos para la Educación, la Ciencia y la Cultura (OEI), Octaedro, 2005, p.97.

FERNÁNDEZ RIUS, Lourdes; BLAZQUEZ GRAF, Norma. Recomendaciones para elaborar políticas de ciencia y tecnología desde una perspectiva de género. Conclusiones del Primer Coloquio Iberoamericano: Diálogo de Saberes y Políticas de Ciencia, Tecnología e Innovación con perspectiva de Género. Cuernavaca Morelos, México, 23-25 de junio de 2015.

Foro Consultivo Científico y Tecnológico. Una Mirada a la ciencia, la tecnología y la innovación con perspectiva de género: hacia un diseño de políticas públicas. Documento de Trabajo. México, noviembre 2013.

HARDING, Sandra. Ciencia y Feminismo. Madrid, Ediciones Morata, 1996.

Hierro Pérez-CAStro, Graciela. Género y empoderamiento, ética y feminismo. In: Bustos ROMERO, O. \& BlAZQUEZ GRAF, N. (coord) Qué dicen las mujeres acerca de la UNAM. Edición Colegio de Académicas Universitarias, Universidad Nacional Autónoma de México, 2003. 
HUYER Sophia Gender and Science and Technology from an International Perspective. Oficina de Ciencia y Tecnología de la Organización de los Estados Americanos y la Comisión Interamericana de la Mujer de las Naciones Unidas. Washington D.C., 2004.

INSTITUTO Nacional de Estadística y Geografía. Mujeres y hombres en México 2014. México, 2015 [www.cedoc.inmujeres.gob.mx/documentos_download/101256.pdf]

LAGARDE, Marcela. Género y feminismo. Desarrollo humano y democracia. Madrid, Ed. horas y HORAS, 1997, p.11.

MAFFÍA, Diana. Sujetos, política y ciudadanía. In: CHAHER, S.; SANTORO, S. (comp.) Las palabras tienen sexo: Introducción a un periodismo con perspectiva de género. Buenos Aires, Artemisa Comunicación Ediciones, 2007a, p.17

MAFFíA, Diana. Epistemología feminista: La subversión semiótica de las mujeres en la ciencia. Revista Venezolana de Estudios de la Mujer, vol.12, n 28, Caracas, junio 2007b.

MEDIDAS para la Inclusión Social y la Equidad en Instituciones de Educación Superior en América Latina [http://www.miseal.net/].

MENDIETA, Angélica. ¿Legitimidad o reconocimiento? Las investigadoras del SNI retos y propuestas. La Biblioteca, México, 2015.

Meza Montes, Lilia; Martínez, Amalia; Xoconostle, Beatriz; PÉrezARMENDARIZ, Elia Martha (eds.) Latinoamericanas en las Ciencias Exactas y de la Vida. Memorias de la II Conferencia Latinoamericana Ciencia Mujer 2006. UNAM, México, D. F., 2009.

Olvera Castillo, Carolina. Mujeres en el Sistema Nacional de Investigadores de la Universidad Autónoma de San Luis Potosí: su acceso, permanencia y promoción. Tesis (Maestría en Políticas Públicas y Género), FLACSO México, Junio, 2015.

PÉREZ-ARMENDARIZ, Elia Martha. Hacia la inclusión de la equidad de Género en la Política de Ciencia y Tecnología en México. Investigación y Ciencia, UAA, 2010, pp.43-56.

PÉREZ-ARMENDARIZ, Elia Martha. Importancia del reconocimiento del campo de ciencia y género por los sistemas de evaluación. In: 
BlAZQUEZ GRAF, Norma (coord.) Evaluación Académica: Sesgos de Género. CEIICH, UNAM/CYTED, México, 2014, pp.371-377.

PÉREZ SEDEÑO, Eulalia. El poder de una ilusión: Ciencia Género y Feminismo. In: Feminismos: del pasado al presente. España, Ediciones Universidad de Salamanca, 2000.

PÉREZ SEDEÑO, Eulalia. La perspectiva del género en Ciencia y Tecnología: innovación y nueva caracterización de las disciplinas. In: Ciencia, Tecnología, Sociedad y cultura en el cambio de siglo. Madrid, Editorial Biblioteca Nueva, 2001, p.46.

PNUD. Conciliación con Co-responsabilidad social en América Latina, ¿cómo avanzar?

PRIMERA Reunión de Ministros y principales representantes en Ciencia y Tecnología, convocada por la OEA y realizada en Lima, Perú, en el 2004.

PRocesos Gráficos. El Salvador, 2010, p.18.

Quintana Nedelcu, Danay. El Sistema de Educación Superior en México. Cuadernos de Trabajo de la Red Mexicana de Ciencia, Tecnología y Género. 2016 [www.redmexciteg.org].

REGLAMENTO del Sistema Nacional de Investigadores. 2013 [www.conacyt.mx].

Reunión Hemisférica de Expertos en Género, Ciencia y Tecnología. Oficina de Ciencia y Tecnología de la Organización de los Estados Americanos y Comisión Interamericana de la Mujer de las Naciones Unidas. Washington, EU, agosto de 2004.

VIRGILÍ, Dalia. Género y Trabajo remunerado. Experiencia grupal de investigación-acción con mujeres y hombres dirigentes del Ministerio del Transporte. Tesis (Maestría en Psicología), Facultad de Psicología, Universidad de La Habana, 2012. 